\title{
Conflicto marital y consumo de drogas en los hijos
}

\author{
Mireia Sanz ${ }^{1-4}$, loseba Iraurgi ${ }^{2-4}$, Ana Martínez-Pampliega ${ }^{3-4}$, Leire Cosgaya ${ }^{3-4}$ \\ 1. Servicio de Prevención de Drogodependencias. Mancomunidad del Txorierri. Bizkaia. \\ 2. Módulo de Asistencia Psicosocial de Rekalde. Bilbao. Bizkaia. \\ 3. Facultad de Psicología. Universidad de Deusto. Bilbao. Bizkaia. \\ 4. AEFFA - Asociación para el Estudio del Funcionamiento Familiar. \\ Enviar correspondencia a:
}

loseba Iraurgi Castillo. Módulo de Asistencia Psicosocial de Rekalde. Camilo Villabaso 24, lonja. 48002 Bilbao. E-mail: iraurgi@euskalnet.net

\section{RESUMEN}

Objetivo.- Se analiza la relación entre el funcionamiento familiar, los conflictos de la pareja y el consumo de drogas por parte de los hijos. Método.- A través de un diseño transversal se ha encuestado a una muestra de 130 jóvenes (72 chicas y 57 chicos; con edades comprendidas entre los 13 y 17 años) y sus respectivas madres. Para la valoración de las variables de interés se ha utilizado una batería de cuestionarios que comprende los siguientes: Consumo de Sustancias (CS), la Escala de Percepción de los Hijos del Conflicto Interparental (CPIC), el Inventario para Hijos del Comportamiento Parental (CRPBI), la Escala de Evaluación de Adaptabilidad y Cohesión Familiar (FACES), el Índice de Satisfacción Matrimonial (ISM) y la Escala de Estabilidad Matrimonial (EEM). Los hijos respondían a los cuatro primeros (CS, CPIC, CRPBI y FACES), mientras sus familiares lo hacían a los tres últimos (FACES, ISM, EEM). Para el análisis de las relaciones entre variables se utilizaron modelos de regresión logística tomando como variable dependiente ser o no ser el hijo consumidor de sustancias. Resultados.- Cuatro dimensiones de conflicto marital (frecuencia: $O R=1,30$; intensidad: $O R=1,56$; inestabilidad: $O R=1,32$; y contenido: $O R=1,33$ ), siete de las ocho dimensiones del CRPBI y la percepción de cohesión familiar por parte de los hijos $(O R=0,51)$ resultaron estadísticamente significativos $(p<0,05)$. No obstante, el control del conjunto de variables mediante un modelo de regresión múltiple ofreció significación estadística para sólo dos de ellas: la percepción de cohesión familiar por parte del hijo/a $(O R=0,59 ; p=0,03)$ y la percepción de control por parte de la madre $(O R=0,69 ; p=0,04)$. Conclusiones.- Los resultados muestran que la presencia de conflicto entre los padres se asocia a una mayor presencia de consumo de sustancias entre los hijos, si bien son necesarias más investigaciones para confirmar estos hallazgos. El estudio sí permite constatar el efecto protector de la cohesión familiar y el control materno percibido por los hijos sobre la probabilidad de consumir drogas por éstos.

Palabras-clave: Consumo de drogas, adolescentes, funcionamiento familiar, conflictos de pareja.

\section{ABSTRACT}

Objective.-We examined the relationship between family functioning, marital conflict and drug use by children. Method.A cross-over design was used in interviewing a sample of 130 young people (72 girls and 57 boys, ranging from 13 to 17 years of age) and their respective mothers. The battery of questionnaires, used to evaluate the variables of interest, comprised: Substance Use (SU), the Children's Perception of Interparental Conflict Scale (CPIC), the Children's Report on Parent Behaviour Inventory (CRPBI), the Family Adaptability and Cohesion Evaluation Scale (FACES), Marital Satisfaction Index (MSI), and the Marital Stability Scale (MSS). The children answered the first four (SU, CPIC, CRPBI and FACES), and their mothers the last three (FACES, MSI and MSS). Logistic regression models were used to analyse the relationship between different variables, taking the child being a substance user or non-user as the dependent variable. Results.- Four dimensions of marital conflict (frequency: $O R=1.30$; intensity: $O R=1.56$; uncertainty: $O R=1.32$; and content: $O R=1.33$ ), seven of the eight $\mathrm{CRPBI}$ dimensions and the family cohesion perceived by children $(O R=0,51)$ were statistically significant $(p<0,05)$. Nevertheless, multiple regression on the group of variables showed statistical significance for only two of them, the children's perception of family cohesion $(O R=0.59 ; p=0.03$ ) and the mothers' perception of control $(O R=0.69 ; p=004)$. Conclusions.- The results show that the presence of conflict between parents is associated with a greater presence of drug use in children; although further research is necessary to confirm these relationships. However, the study does point to family cohesion, and children's perception of control by the mother as having a protective effect on the probability of drug use by the former.

Keywords: Drug use, adolescent, family relations, marital conflict. 


\section{INTRODUCCIÓN}

$\mathbf{L}$ a familia constituye el entorno social con más influencia en la formación y desarrollo de niños y jóvenes. Aunque otros contextos sociales (grupo de iguales, escuela, medios de comunicación...) son importantes, pasan normalmente por el tamiz de la familia pudiendo amplificar o disminuir los efectos de ésta. En consecuencia, los análisis de los factores de riesgo en relación al consumo de drogas tienen a la familia como uno de los principales objetivos de investigación $n^{1-6}$.

Para algunos autores el consumo de drogas en los padres puede propiciar el consumo de las mismas en los hijos ${ }^{7-10}$. Otra vertiente, sin embargo, defiende que es la existencia de problemas de relación en la familia y sus consecuencias en el clima familiar lo que puede llegar a proporcionar el consumo de drogas en los hijos $^{11-13}$.

Las variables familiares de mayor interés en relación al campo de las drogodependencias han sido la supervisión parental / tipo de disciplina ${ }^{14-15}$; el apego / cercanía ${ }^{16-17}$; la comunicación familiar / cohesión ${ }^{18-20}$ y el conflicto marital ${ }^{21-24}$.

Respecto a esta última variable, son frecuentes las investigaciones que apuntan a la existencia de un nexo entre el conflicto marital y las conductas problemáticas por parte de los hijos ${ }^{25-31}$. Los estudios indican que la intensidad y la frecuencia del conflicto, el estilo del conflicto, su manera de resolución y la presencia de intermediarios a fin de aminorar los efectos del conflicto, son los más importantes predictores para la adaptación de los hijos ${ }^{32}$. Sin embargo, son escasos los estudios que han vinculado las relaciones matrimoniales con el consumo de drogas. Entre ellos podemos destacar a Malkus ${ }^{33}$, quien al comparar dinámicas familiares entre familias con un miembro adolescente drogodependiente y familias sin ningún miembro drogodependiente puso de manifiesto que los adolescentes que perciben a sus padres como parejas "felices" se encuentran en el último de los grupos mencionados. Similares resultados fueron encontrados por Ruiz, Lozano y Polaino ${ }^{34}$ quienes afirman que el grado de estabilidad de la pareja de padres influye directamente en los patrones de consumo de alcohol y drogas ilegales en los hijos.

Por otra parte, Muñoz-Rivas y Graña ${ }^{35}$ analizaron la influencia de determinadas variables familiares en el consumo de drogas por parte de los adolescentes. Los resultados mostraron que una de las dimensiones que predecían mayor riesgo de uso de sustancias por parte del adolescente era la existencia de conflictos entre los padres.

Entre los mecanismos explicativos de la relación entre el conflicto marital y el consumo de drogas por parte de los hijos se hallan variables familiares relevan- tes como la disciplina, la hostilidad y el afecto. Esta vía de influencia del conflicto matrimonial en el consumo de drogas postula un efecto indirecto, es decir los conflictos matrimoniales actuarían sobre el consumo de sustancias por parte de hijos e hijas a través del aumento de relaciones coercitivas de los padres y por un deterioro en las prácticas de crianza. Los roles que desempeñan los padres con los hijos se verían afectados por el conflicto matrimonial en estas áreas ${ }^{36-37}$.

Nuestro objetivo en este estudio es explorar y comprender la relación entre el funcionamiento familiar (valorado a través del conflicto marital, las relaciones padres-hijos y la cohesión y adaptabilidad familiar) y el consumo de drogas en los adolescentes.

\section{MÉTODO}

El presente estudio se haya encuadrado dentro de un estudio más amplio cuyo objetivo era valorar y analizar las consecuencias del conflicto marital y la separación de los padres, y por ende de la familia, en la salud y bienestar de los hijos.

\section{Muestra}

Partiendo del censo de alumnos prematriculados para el curso 2003-2004 en los niveles de $3^{\circ}$ y $4^{\circ}$ de Enseñanza Secundaria Obligatoria (ESO) en Bizkaia, se seleccionaron al azar los centros y aulas que conformarían la muestra total. Se buscó la representatividad de la población teniendo en cuenta los siguientes criterios: comarcas, municipios, modelos lingüísticos, curso académico y titularidad del centro. Se estableció contacto con los centros seleccionados para explicarles el objetivo del estudio y pedir su colaboración. Accedieron a participar en el estudio 19 centros, y un total de 853 alumnos(as).

En el estudio que aquí se presenta han participado 130 díadas de estudiantes y sus respectivas madres. De los 130 alumnos, un $55,4 \%(n=72)$ eran chicas y un $43,8 \%(n=57)$ chicos. La edad media ha resultado de 14,6 (DT= 0,7) años, dentro de un recorrido de edades de entre 13 y 17 años, siendo los grupos más representativos los de 14 y 15 años. Respecto al grado académico, un 54,6\% cursaba tercero de ESO y un $45,4 \%$ cuarto curso. Para cada alumno participante se contactó con un miembro familiar que en todos los casos fue la madre. En 99 casos estaban casadas $(76,7 \%)$, en 18 casos $(18 \%)$ estaban separadas o divorciadas, 5 eran madres solteras y 7 habían enviudado. Estas tres últimas situaciones comportan un cuidado de la familia a cargo de la madre, y constituyen el $23,3 \%$ de los casos $(n=30)$. En 100 casos $(76,9 \%)$ de los alumnos entrevistados viven con sus padres y/u otros miembros familiares, $19(14,6 \%)$ viven con su madre, en 11 casos $(8,5 \%)$ con ésta y/u otros miem- 
bros familiares. En 27 díadas (20,8\%) la descendencia estaba constituida por un solo miembro, es decir, en 27 casos son familias con un/a solo/a hijo/a; el caso más representativo han sido las familias con dos hijos ( $n=67 ; 51,5 \%$ ) y con más de tres hijos han resultado un $27,7 \%$ de los casos $(n=36)$.

\section{Procedimiento}

Seleccionados los centros educativos la investigadora principal del proyecto contactó con los directores y con las asociaciones de padres de alumnos (APAs) para explicarles el objetivo del estudio y pedir su colaboración. Un equipo de campo formado por dos personas, con entrenamiento sobre las características y administración del cuestionario, acudía al centro educativo en fecha y hora previamente concertadas. El cuestionario era administrado colectivamente en las aulas seleccionadas con presencia física del equipo de campo, para solucionar las posibles dudas en su contestación, y ausencia del profesorado. Los cuestionarios eran anónimos, el alumnado podía negarse a contestarlo y se garantizaba la confidencialidad de la información en ellos contenida.

Con respecto a la selección de las madres se pidió su colaboración a través de la información ofrecida a los centros y las APAs. Se solicitó un aula y una fecha apropiada en el centro para atender a los familiares que acudieran. En esta reunión se informó de las características y objetivos de la investigación y, posteriormente, tuvo lugar la pasación de cuestionarios. Además de la reunión en el centro, se ofreció también la opción a todas las madres que no pudieran acudir a la cita establecida, de acudir en otro momento y lugar a concertar. Accedieron a participar un total de 130 familiares. Para relacionar a los participantes familiares con sus respectivos hijos se utilizó una clave común que podían utilizar ambos miembros pero que impedía la identificación concreta del adolescente (dos primeras letras del nombre del adolescente más el día y mes de la fecha de nacimiento).

\section{Instrumentos}

La información ha sido recogida a través de un cuestionario respondido por los propios alumnos y los familiares, en el cual se incorporaron una batería de escalas que se describen a continuación.

CPIC - Escala de Percepción de los Hijos del Conflicto Interparental (Children's Perception of Interparental Conflict Scale) ${ }^{38}$

Fue desarrollado por Grych y colaboradores ${ }^{38-39}$ y permite valorar los puntos de vista de los hijos acerca de varios aspectos del conflicto marital. Valora nueve dimensiones teóricas que atañen tanto a las características del conflicto [a) frecuencia, b) estabilidad, c) intensidad, d) resolución], como la vivencia o evaluación del conflicto por el niño/a [e) contenido, f) autoculpa], así como a la percepción de amenazas y afrontamiento [g) sentimientos de amenaza, h) eficacia de afrontamiento, i) triangulación]. En nuestro estudio se ha utilizado la adaptación de 36 ítems realizada por el grupo de Martínez-Pampliega ${ }^{40}$. Cada una de las 9 dimensiones teóricas es valorada por cuatro ítems de respuesta tipo likert de cinco puntos (1 a 5). Para cada dimensión se suman los valores de los ítems correspondientes y la puntuación total del CPIC se obtiene a partir de la suma de las nueve dimensiones. Una mayor puntuación corresponde con una mayor expresión del conflicto marital en cada una de las nueve dimensiones valoradas. La versión de 36 ítems del CPIC utilizada mostró en su estudio de adaptación y validación un buen comportamiento psicométrico ${ }^{40}$, ofreciendo valores de fiabilidad entre 0,65 y 0,82 en las diferentes dimensiones y siendo el valor alpha de Cronbach para la escala total de 0,82 . Este instrumento, al igual que el siguiente, fue aplicado exclusivamente a los adolescentes.

CRPBI - Inventario para Hijos del Comportamiento Parental (Child's Report on Parental Behavior Inventory) ${ }^{41}$

El CRPBI es un cuestionario diseñado para evaluar la percepción de los hijos respecto al comportamiento de los padres en referencia a la aceptación, disciplina y hostilidad parental. Han sido desarrolladas varias versiones de este instrumento. Nosotros utilizaremos la adaptada por el grupo de Mestre ${ }^{42}$, compuesta por 52 ítems organizados en 4 subescalas (amor, control, hostilidad y autonomía) cada una de las cuales está valorada respecto a ambas figuras parentales.

Los ítems plantean diferentes situaciones propias de la vida y educación familiar a las que el adolescente debe contestar en una escala de tres puntos (1-3), según el grado de acuerdo con el planteamiento del ítem. Se obtienen, respecto a cada figura parental, cuatro puntuaciones derivadas del sumatorio de ítems que componen cada subescala. Una mayor puntuación en cada una de estas dimensiones expresaría, desde la percepción del adolescente, un mayor grado de expresión del progenitor en la cualidad valorada.

Los valores de fiabilidad alcanzados por el instrumento muestran valores de consistencia interna desiguales, bajos en el caso de la dimensión de autonomía (alphas de 0,34 y 0,48; en el caso de la subescala referente a la madre y al padre, respectivamente), moderados-bajos en la dimensión Control $(0,58$ y 0,59), y moderados altos en la dimensiones de amor $(0,78$ y $0,84)$ y Hostilidad $(0,70$ y 0,71$)$.

FACES - Escalas de Evaluación de Adaptabilidad y Cohesión Familiar (Family Adaptability and Cohesion Evaluation Scale).

EI FACES es un instrumento dirigido a evaluar la cohesión y la adaptabilidad del funcionamiento familiar, las cuales constituyen las dos dimensiones cen- 
trales del modelo Circumplejo ${ }^{43-46}$. La cohesión es el vínculo emocional que los miembros de un sistema (la familia, en nuestro caso) tienen entre sí y la adaptabilidad sería la habilidad de dicho sistema para cambiar. En este estudio hemos empleado la versión FACES-30$\mathrm{Esp}^{40}$, que cuenta con una buena fiabilidad tanto en la escala de cohesión como de adaptabilidad (alpha: 0,78 y 0,70$)$, respectivamente. Cada una de las dimensiones consta de 15 ítems de formato likert de 5 puntos (1-5) que son agregados para ofrecer una puntuación global, de modo que una mayor puntuación expresaría una mayor presencia de la característica que se evalúa, cohesión o adaptabilidad.

Este instrumento fue aplicado a los adolescentes y familiares, encontrándose una fiabilidad aceptable en ambas submuestras: alpha: 0,89 y 0,87 para la aplicación al adolescente, y 0,86 y 0,72 para la aplicación al familiar.

\section{Índice de Satisfacción Matrimonial ${ }^{47}$.}

Es un instrumento diseñado para medir el grado, severidad o magnitud de un problema que el/la esposo/a tenga en su relación de pareja ${ }^{47}$. Actualmente es uno de los instrumentos incluidos en las escalas de evaluación WALMYR ${ }^{48}$. Consta de 25 ítems tipo likert (rango de respuesta de 1 a 6) cuyos valores se agregan para dar una única puntuación del instrumento, de forma que una mayor puntuación indicaría un mayor grado de satisfacción matrimonial. En el estudio de adaptación y validación a nuestro medio ${ }^{40}$ se observó un único factor que explicaba el 63,8\% de la varianza y una alta consistencia interna (alpha de Cronbach: 0,97). La fiabilidad observada por la muestra de familiares participantes en el estudio también fue alta, alcanzando un valor de 0,91.

\section{Escala de Estabilidad Matrimonial ${ }^{49}$.}

Aplicado a los familiares, es un instrumento fiable y valido para predecir el divorcio de una pareja ${ }^{49}$. Consta de cinco ítems e incluye los siguientes aspectos: pensar en el divorcio, hablar con el cónyuge sobre la obtención de divorcio, hablar con otras personas significativas, encuentros con profesionales o consejeros y situaciones de separación física con la pareja. Cada uno de los ítems es respondido a través de una escala de intensidad de cuatro puntos, cuyos valores se agregan para ofrecer una puntuación total. En la escala original una mayor puntuación indicaría un mayor grado de inestabilidad, pero en nuestro estudio para permitir una interpretación acorde con otras escalas de funcionamiento familiar (Escala de satisfacción y FACES) se han invertido los valores de forma que una mayor puntuación representaría un mayor grado de estabilidad matrimonial.

Tanto en el estudio original ${ }^{49}$ como en la adaptación a nuestro medio ${ }^{40}$ se han obtenido altos valores de consistencia interna (alpha de Cronbach de 0,93 y 0,90 respectivamente). Asimismo, en el estudio de adaptación se halló unidimensionalidad en un análisis de com- ponentes principales, Ilegando a explicar el 72,2\% de la varianza.

\section{Consumo de drogas}

Fue valorado exclusivamente entre los adolescentes. El cuestionario sobre consumo utilizado en la presente investigación es una adaptación del instrumento empleado en el estudio "Usos y consumos de drogas entre la población de 16 a 25 años". Este cuestionario consta de 76 preguntas y está basado en dos fuentes: el cuestionario Drogas y Escuela $V^{11}$ y el cuestionario Euskadi y Drogas $1998^{50}$

Como indicadores del consumo de sustancias entre los adolescentes se han considerado aquellos que han mostrado mayor relevancia y discriminación. En concreto, se han elegido cinco variables, a saber: 1) la frecuencia de consumo de tabaco, 2) la frecuencia de consumo de alcohol, 3) la frecuencia de borracheras, 4) la frecuencia de consumo de porros, y 5) el número de drogas consumidas. A partir de estas cinco variables se ha generado un indicador dicotómico que adopta las categorías de 'consumidor' o 'no consumidor', siendo considerado como 'consumidor' aquel adolescente que 1) fuma habitualmente al menos un cigarrillo al día, 2) bebe de vez en cuando, los fines de semana o a diario, 3) se ha emborrachado alguna vez, 4) ha fumado cannabis en más de cinco ocasiones, o 5) ha consumido algún otro tipo de sustancia. Este indicador no pretende ser un reflejo de un consumo problemático, sino de una mayor frecuencia de contacto con las sustancias de abuso que pudieran conducir a la generación de un hábito de consumo.

\section{Análisis estadísticos}

Dado que no existen datos normativos de la población española para la mayoría de los instrumentos utilizados, se ha procedido a expresar el conjunto de las mismas bajo una misma escala de medida. Las puntuaciones globales de todos los instrumentos evaluados, a excepción de las variables que valoran el consumo de sustancias, fueron transformadas a escala decimal a través del siguiente algoritmo: puntuación obtenida menos la puntuación mínima posible de la escala multiplicado por la razón entre diez y la puntuación máxima posible de la escala; [por ejemplo, en el caso de la satisfacción familiar la puntuación para una persona que ha obtenido un valor suma de 89 será: (89-25) x $(10 / 125)=5,12]$. De este modo, la valoración de las características evaluadas seguiría una interpretación ampliamente conocida (cero equivale a ausencia de la característica y diez al máximo posible de expresión) y permite, asimismo, la comparación de la puntuación en una escala respecto al obtenido en las demás.

Se ha analizado la fiabilidad de las escalas utilizadas a través del coeficiente alpha de Cronbach $(\alpha)$, ofreciendo una estimación de la consistencia interna de las mismas. Para la descripción de la muestra se ha utilizado el recuento de frecuencias y porcentajes para el 
caso de variables cualitativas, y en el caso de variables continuas se ha recurrido a las medidas de tendencia central (Media - M), dispersión (Desviación Típica - DT) y valores mínimo y máximo (Min-Max). Se ofrecen los valores medios y de dispersión el las escalas utilizadas para expresar el nivel de funcionamiento familiar y consumo de drogas.

El estudio de asociación entre el funcionamiento familiar y el consumo de drogas por parte de los hijos se ha realizado poniendo en relación el conjunto de variables que expresan el funcionamiento familiar con el indicador 'consumidor'. No se ha contemplado la asociación de las primeras con las variables de consumo de sustancias a través de análisis de correlación, por ejemplo, en la medida que estas últimas no satisfacen las condiciones de aplicación (distribuciones asimétricas no normales).

Dado que el indicador 'consumidor' es una variable dicotómica, se ha calculado la Odds Ratio (OR) por considerar que es el coeficiente más adecuado a este tipo de variable. Para su cálculo se ha utilizado la técnica de regresión logística simple que nos proporciona un valor de OR crudo, su intervalo de confianza al 95\% y la prueba de Wald asociada con su nivel de signifi- cación ${ }^{51}$. Las variables de funcionamiento familiar que resultaron significativas a nivel bivariado fueron incluidas en un modelo de regresión logística multivariado en aras a estimar el efecto conjunto, obteniéndose, en este caso, las OR ajustadas. Para ello se ha utilizado una estrategia basada en la introducción condicional por pasos del conjunto de variables seleccionadas con un criterio de inclusión (PIN) de 0,05 y un valor probabilidad de exclusión (POUT) de 0,1.

Para valorar los resultados se ha elegido un nivel de significación de $p<0,05$, para un intervalo de confianza del 95\% (IC 95\%). En general, estos análisis de datos han de considerarse como exploratorios. Los análisis han sido realizados con el Programa SPSS para Windows en su versión 10

\section{RESULTADOS}

\section{Funcionamiento familiar}

En la tabla 1 se recogen las características descriptivas y fiabilidad alcanzadas por el conjunto de instrumentos que dan muestra del funcionamiento familiar, valorados tanto por los hijos como por las madres. Los índices de fiabilidad alcanzados por el conjunto

\section{Tabla 1.- Valoración de los componentes familiares. Características descriptivas y fiabilidad.}

\begin{tabular}{|c|c|c|c|}
\hline & Fiabilidad (alpha Cronbach) & Media & Desviación típica \\
\hline \multicolumn{4}{|l|}{ CPIC - CONFLICTO MARITAL (valoración hijo/a) } \\
\hline Intensidad & 0,98 & 3,08 & 1,03 \\
\hline Frecuencia & 0,81 & 1,38 & 1,84 \\
\hline Inestabilidad & 0,78 & 1,09 & 1,62 \\
\hline Irresolución & 0,82 & 1,43 & 1,79 \\
\hline Contenido & 0,82 & 1,54 & 1,74 \\
\hline Ineficacia de afrontamiento & 0,63 & 2,41 & 1,81 \\
\hline Amenaza percibida & 0,84 & 1,64 & 2,01 \\
\hline Triangulación & 0,60 & 1,06 & 1,38 \\
\hline Autoculpa & 0,85 & 1,06 & 1,58 \\
\hline Total & 0,91 & 1,58 & 1,14 \\
\hline \multicolumn{4}{|l|}{ CRPBI - COMPORTAMIENTO PARENTAL } \\
\hline \multicolumn{4}{|l|}{ REFERENTE A LA MADRE (valoración hijo/a) } \\
\hline Amor & 0,78 & 5,62 & 2,01 \\
\hline Hostilidad & 0,76 & 3,44 & 2,21 \\
\hline Control & 0,73 & 6,89 & 2,01 \\
\hline Autonomía & 0,58 & 2,41 & 2,53 \\
\hline \multicolumn{4}{|l|}{ REFERENTE AL PADRE (valoración hijo/a) } \\
\hline Amor & 0,73 & 6,58 & 1,91 \\
\hline Hostilidad & 0,74 & 2,42 & 1,88 \\
\hline Control & 0,78 & 3,04 & 2,29 \\
\hline Autonomía & 0,56 & 3,10 & 2,47 \\
\hline \multicolumn{4}{|l|}{ FACES - FUNCIONAMIENTO FAMILIAR (valoración hijo/a) } \\
\hline Cohesión & 0,89 & 6,66 & 1,40 \\
\hline Adaptabilidad & 0,87 & 6,06 & 1,29 \\
\hline \multicolumn{4}{|l|}{ (valoración madre) } \\
\hline Cohesión & 0,86 & 7,74 & 1,19 \\
\hline Adaptabilidad & 0,72 & 6,89 & 0,97 \\
\hline ESTABILIDAD MATRIMONIAL (valoración madre) & 0,83 & 9,22 & 1,27 \\
\hline SATISFACCIÓN MATRIMONIAL (valoración madre) & 0,96 & 7,38 & 2,44 \\
\hline
\end{tabular}


de instrumentos están por encima del valor 0,70, a excepción de las dimensiones ineficacia de afrontamiento $(\alpha=0,63)$ y triangulación $(\alpha=0,60)$ del CPIC, y las dimensiones de autonomía del CRPBI, tanto atendiendo a la percepción hacia la madre $(\alpha=0,58)$ como hacia el padre $(\alpha=0,56)$.

La puntuación media más alta de las dimensiones del CPIC es de 3,08 sobre un máximo de 10 (dimensión de intensidad), indicándose en conjunto un bajo grado de conflicto marital percibido por los hijos. Las valoraciones que realizan los hijos respecto al compor- tamiento que realizan sus padres en las áreas exploradas por el CRPBI se hallan por encima de 5 en tres áreas [relación de amor de la madre $(M=5,62)$ y del padre $(M=6,58)$, y relación de control por parte de la madre $(M=6,89)]$; todas las demás áreas muestran puntuaciones medias por debajo del valor 3,5 sobre 10. La valoración de la cohesión y adaptabilidad familiar, estimada tanto por hijos como por las madres, alcanzan valores superiores a 6 puntos sobre 10 , y en el caso de la estabilidad y satisfacción matrimonial valorada por la madre se obtienen valores de 9,2 y 7,4 puntos sobre 10, respectivamente.

\section{Criterios de valoración del consumo de sustancias \\ Tabaco: Fumar habitualmente al menos un cigarrillo al día Alcohol: Beber de vez en cuando, los fines de semana o diario \\ Borracheras: Haberse emborrachado alguna vez \\ Porros: Haber fumado cannabis en más de cinco ocasiones Drogas: Haber consumido alguna droga ilegal distinta del cannabis Consumidor: Aquel que cumple alguno de los criterios previos}

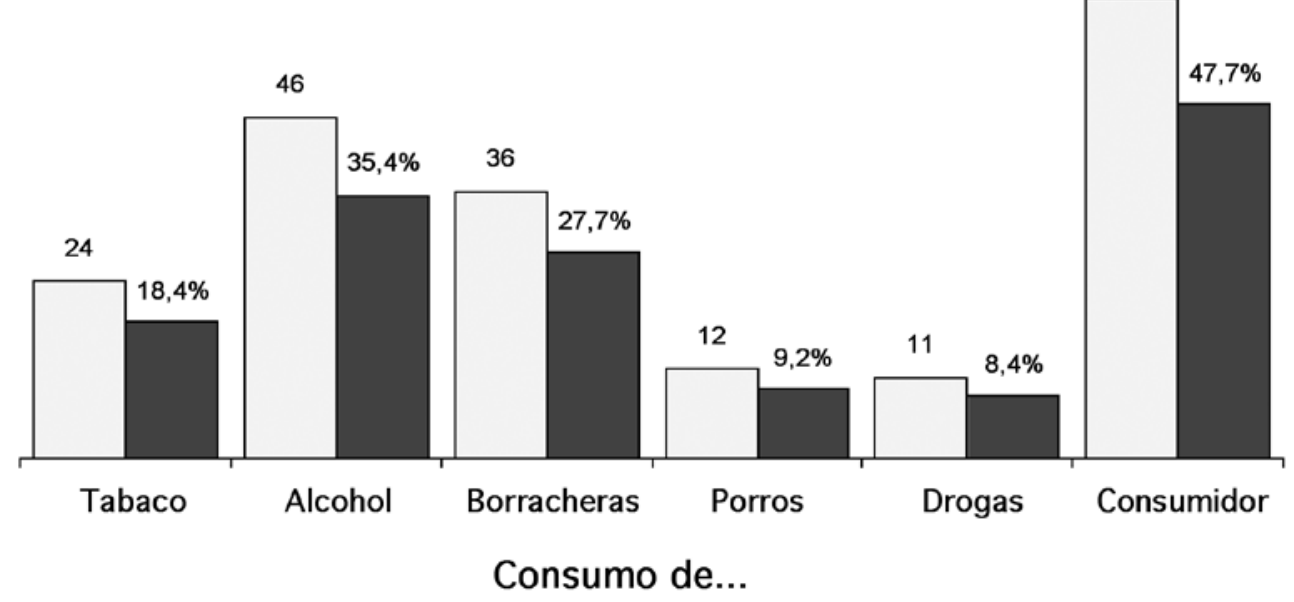

Figura 1. Indicadores de consumo de sustancias y prevalencia de consumidores.

\section{Consumo de sustancias}

Se ha creado un indicador de consumo de drogas a partir de cinco criterios de prevalencia de consumo de diversas sustancias realizado por los adolescentes, y cuyos resultados aparecen en la figura 1. La mayor prevalencia de casos de consumo se localiza en el uso de alcohol $(35,4 \%)$, habiéndose emborrachado alguna vez un $27,7 \%$ de los casos. Un $18,4 \%$ de los adolescentes es fumador/a durante el fin de semana y menos del $10 \%$ han fumado porros $(9,2 \%)$ o han consumido alguna vez alguna droga ilegal $(8,4 \%)$. La conjunción de estas prevalencias nos muestra como 62 de los 130 adolescentes entrevistados (un 47,7\%) han mostrado signos de contacto con sustancias o frecuencia de consumo que les lleva a ser clasificados como consumidores.

\section{Variables familiares asociadas a ser 'consumidor'}

En la tabla 2 se recogen los resultados de asociación bivariada entre cada componente familiar y ser clasificado en la categoría de consumidor a través de análisis de regresión logística simple, presentándose las OR crudas, el intervalo de confianza del 95\% de la OR y el grado de significación estadística vinculado a la prueba de wald. Cuatro variables del CPIC (intensidad, frecuencia, inestabilidad y contenido), seis del CRPBI (relaciones de amor, hostilidad, control y autonomía por parta de la madre, y relaciones de amor y control del padre) y una del FACES (cohesión percibida por el hijo) han mostrado asociación estadísticamente significativa con ser consumidor.

La Tabla 3 recoge el modelo de regresión logística múltiple con introducción consecutiva de variables de 
Tabla 2.- Asociaciones entre el funcionamiento familiar y la variable 'consumidor'.

Análisis de regresión logística simple'.

\begin{tabular}{|c|c|c|c|c|}
\hline & OR & IC 95\% & Wald & $\mathbf{p}$ \\
\hline \multicolumn{5}{|l|}{ CPIC - CONFLICTO MARITAL (valoración hijo/a) } \\
\hline Intensidad & 1,56 * & $1,06-2,32$ & 5,00 & 0,025 \\
\hline Frecuencia & $1,30 *$ & $1,05-1,63$ & 5,66 & 0,017 \\
\hline Inestabilidad & $1,32 *$ & $1,03-1,70$ & 4,87 & 0,027 \\
\hline Irresolución & 1,16 & $0,93-1,44$ & 1,86 & 0,171 \\
\hline Contenido & $1,33 *$ & $1,06-1,68$ & 6,04 & 0,014 \\
\hline Ineficacia de afrontamiento & 0,96 & $0,78-1,18$ & 0,13 & 0,711 \\
\hline Amenaza percibida & 1,09 & $0,90-1,32$ & 0,81 & 0,365 \\
\hline Triangulación & 1,09 & $0,83-1,44$ & 0,44 & 0,507 \\
\hline Autoculpa & 1,19 & $0,93-1,53$ & 2,06 & 0,151 \\
\hline Total & 1,37 & $0,95-1,96$ & 2,90 & 0,088 \\
\hline \multicolumn{5}{|l|}{ CRPBI - COMPORTAMIENTO PARENTAL } \\
\hline \multicolumn{5}{|l|}{ REFERENTE A LA MADRE(valoración hijo/a) } \\
\hline Amor & $1,29 *$ & $1,06-1,58$ & 6,52 & 0,010 \\
\hline Hostilidad & $1,24 *$ & $1,05-1,47$ & 6,14 & 0,013 \\
\hline Control & $0,75 * *$ & $0,62-0,92$ & 7,71 & 0,005 \\
\hline Autonomía & $1,15 *$ & $1,01-1,34$ & 3,73 & 0,049 \\
\hline \multicolumn{5}{|l|}{ REFERENTE AL PADRE (valoración hijo/a) } \\
\hline Amor & $0,69 * *$ & $0,55-0,87$ & 9,38 & 0,002 \\
\hline Hostilidad & 1,20 & $0,97-1,48$ & 2,90 & 0,088 \\
\hline Control & $1,21 *$ & $1,01-1,43$ & 4,47 & 0,034 \\
\hline Autonomía & 1,00 & $0,85-1,17$ & 0,01 & 0,972 \\
\hline \multicolumn{5}{|l|}{ FACES - FUNCIONAMIENTO FAMILIAR (valoración hijo/a) } \\
\hline Cohesión & $0,51 * * *$ & $0,35-0,74$ & 12,93 & 0,000 \\
\hline Adaptabilidad & 0,74 & $0,54-1,02$ & 3,22 & 0,072 \\
\hline \multicolumn{5}{|l|}{ (valoración madre) } \\
\hline Cohesión & 0,78 & $0,57-1,07$ & 2,28 & 0,130 \\
\hline Adaptabilidad & 0,77 & $0,52-1,14$ & 1,67 & 0,196 \\
\hline ESTABILIDAD MATRIMONIAL (valoración madre) & 0,86 & $0,62-1,19$ & 0,76 & 0,381 \\
\hline SATISFACCIÓN MATRIMONIAL (valoración madre) & 0,89 & $0,76-1,05$ & 1,62 & 0,202 \\
\hline
\end{tabular}

1.- Se realizan 24 regresiones logísticas simples, una por cada variable analizada. OR: Odds Ratio $>1=$ factor de riesgo; $<1=$ factor protector

Tabla 3.- Análisis de regresión logística multivariada (Método Stepwise)

\begin{tabular}{|c|c|c|c|c|}
\hline & \multicolumn{2}{|c|}{ Paso 1} & \multicolumn{2}{|c|}{ Paso 2} \\
\hline & OR (IC 95\%) & Wald (p) & OR (IC 95\%) & Wald (p) \\
\hline Cohesión (valoración del hijo/a) & $\begin{array}{c}0,50 \\
33-078\end{array}$ & 9,60 & $\begin{array}{c}0,59 \\
37-05)\end{array}$ & $\begin{array}{c}4,70 \\
(0,030)\end{array}$ \\
\hline Percepción de Control por la madre & & & $\begin{array}{c}0,69 \\
(0,48-0,99)\end{array}$ & $\begin{array}{c}4,03 \\
(0,045)\end{array}$ \\
\hline Pruebas de idoneidad del modelo & $\begin{array}{r}-2 L L=81,59 ; \\
p<0,0\end{array}$ & $=12,62$ & $\begin{array}{r}-2 \mathrm{LL}=77,32 \\
p<0,0\end{array}$ & $=16,90$ \\
\hline
\end{tabular}

OR: Odds Ratio $>1$ = factor de riesgo; $<1$ = factor protector

forma condicional. Ha proporcionado una solución en dos pasos con criterios de idoneidad estadísticamente significativos. El primer paso incluye la variable cohesión percibida por el hijo con un claro efecto protector $(\mathrm{OR}=0,50$; IC $95 \%=0,33-0,78)$. En el segundo paso se incorpora la relación de control de la madre, también con un efecto protector $(\mathrm{OR}=0,69$; IC $95 \%=$ $0,48-0,99)$, reduciéndose ligeramente el efecto de la variable cohesión $(O R=0,59$; IC 95\%=0,37-0,95). Ninguna de las restantes variables que resultaron significativas en los modelos simples han podido incorporarse en el modelo multivariado. 


\section{DISCUSIÓN}

El objetivo propuesto en este estudio pretendía explorar la relación entre diversas variables de funcionamiento familiar y el consumo de drogas en los y las adolescentes. Entre las variables de funcionamiento familiar se había puesto especial interés, por haber sido tratado con escasa frecuencia en la investigación sobre el área, en el efecto del conflicto marital sobre los consumos sustancias por parte de los hijos. Este objetivo se ha visto respaldado parcialmente, ya que si bien se han observado asociaciones estadísticamente significativas entre esta variable de interés y la variable resultado, no ha mostrado este mismo efecto cuando ha sido controlada en modelos multivariados. Sí han mostrado un efecto más claro variables de funcionamiento familiar, como es la cohesión y un estilo de relación de control de la madre para con sus hijos, en ambos casos con un efecto protector: los adolescentes que perciben mayor cohesión entre los miembros de su familia y una labor de control por parte de su madre son los que presentan una menor probabilidad de consumir drogas.

Los resultados observados han de considerarse como exploratorios, como es propio de todo estudio de corte transversal donde las asociaciones entre las variables, si bien pueden mostrar correlaciones significativas, no permiten inducir líneas de causalidad. Añadida a esta limitación, el tamaño de la muestra no es lo suficientemente grande para permitir reducir el error de medida, por lo que los modelos multivariados tienden a ofrecer estimaciones limitadas. Por todo ello, hemos de ser prudentes en nuestras conclusiones, si bien se abre la posibilidad de comentar los resultados obtenidos en aras a establecer algunas hipótesis de trabajo.

En primer lugar, hemos de observar que la muestra estudiada se caracterizaría por una estructuración y funcionamiento familiar adecuado y positivo; en general, son familias funcionales. Respecto al consumo de drogas, un $47,7 \%$ han sido clasificados como 'consumidores', pero no ha de considerárseles bajo la connotación negativa del término. Esta clasificación ha sido meramente operativa para permitir los análisis de asociación, y en ningún caso han de considerarse como abusadores o consumidores de riesgo. A este respecto, la mayor contribución al porcentaje de adolescentes clasificados como consumidores en este estudio han procedido de las variables relacionadas con el consumo de alcohol, siendo relativamente bajas las prevalencias de consumidores de drogas ilegales. De hecho, cuando observamos las frecuencias y cantidades de consumo tienden a estar ligeramente por debajo de las prevalencias observadas en su población de referencia ${ }^{52}$. Esta mayor funcionalidad familiar, con un bajo nivel de percepción de conflicto marital por parte de los hijos, y un consumo de sustancias mode- rado podría estar en la base de las limitaciones encontradas para probar la hipótesis de nuestro estudio. Probablemente el acceso a muestras con alto conflicto marital pudieran ofrecer relaciones más fuertes con el consumo de drogas de los hijos, pero técnica y deontológicamente acceder a este tipo de muestras es una labor complicada.

Otro hallazgo de interés de nuestro estudio radica en el hecho de que son las variables que implican una valoración del hijo/a las que han mostrado mayor grado de asociación estadística en comparación con las variables que implican la valoración de la madre. Ello permite sugerir que lo que realmente importa en el análisis de las variables de consumo entre los adolescentes es su percepción de las dinámicas familiares. Como señala el grupo de Grych $^{38}$ el conflicto matrimonial no es una variable unidimensional, y junto a la consideración de las propiedades del conflicto (forma en que se expresa el conflicto, la frecuencia y duración de la situación, etc.) es fundamental tener en cuenta la vivencia subjetiva del conflicto por parte del hijo.

Aunque son numerosas las investigaciones que han relacionado el funcionamiento familiar y el consumo de drogas por parte de los hijos, son muy escasas las que han relacionado conflicto marital con el consumo ${ }^{33-35}$. En este sentido, algunos autores ${ }^{53-54}$ confirman que los problemas de pareja, tienen un impacto negativo en las pautas de crianza, facilitando la aparición de comportamientos perturbadores en los hijos. Si bien las variables específicas que circunscriben el conflicto marital no han mostrado un efecto con significación estadística en el modelo multivariado probado en este estudio, si lo han ofrecido cuando los análisis de asociación se establecían de forma bivariada. Las dimensiones del conflicto entre padres que han mostrado significación estadística y en todos los casos se presentan como factores de riesgo son la intensidad $(\mathrm{OR}=1,56)$, el hecho de que el hijo se perciba como motivo del conflicto de los padres -contenido- (OR= $1,33)$, la inestabilidad de la relación marital $(O R=1,32)$ y la frecuencia de los conflictos $(O R=1,30)$. En general, el conflicto marital percibido por el hijo muestra un efecto estadísticamente tendencial, no significativo, sobre la probabilidad de que éste sea clasificado como 'consumidor', pero resulta un efecto que alcanza una magnitud considerable $(\mathrm{OR}=1,37)$. A este respecto, cabe apreciar dos matizaciones. Una primera, técnica, que valores de OR por encima de 1,25 ó por debajo de 0,8 representan un efecto (impacto) destacable ${ }^{51}$; y otra segunda, más conceptual, que en el análisis de resultados es conveniente no sólo destacar lo 'estadísticamente relevante' sino también lo 'clínicamente (o teóricamente) importante ${ }^{55}$. Desde nuestro punto de vista, los resultados obtenidos no nos permiten aseverar con firmeza la relación de causalidad entre el conflicto marital y el consumo de drogas en los hijos, pero nos abre la perspectiva de que ambos fenómenos pue- 
den estar asociados en mayor medida que lo que este estudio ha permitido constatar. Bien el conflicto marital podría influir en el consumo de sustancias de los hijos, a través de mecanismos no explorados en este trabajo, o bien el consumo de sustancias por parte de los hijos pudiera motivar el conflicto entre los padres. Desde nuestro punto de vista, parece que es la primera línea de explicaciones la más plausible, puesto que el grupo de consumidores de drogas en este estudio se encontraba en una fase incipiente del consumo, por lo que es bastante improbable que los conflictos familiares puedan estar precedidos por problemas debidos al uso de drogas. No obstante, esta última hipótesis precisaría de verificación a través de estudios de tipo longitudinal.

En cualquier caso, si bien partimos de unos limitados resultados en cuanto a la relación de asociación estadística entre el conflicto marital y el consumo de drogas entre los hijos e hijas, consideramos que la magnitud del efecto encontrado entre ambas variables permite abrir el debate y la investigación sobre esta área.

\section{AGRADECIMIENTOS}

Estudio financiado por la Dirección de Drogodependencias de Gobierno Vasco dentro del programa de Ayuda a la Investigación en Materia de Drogodepedencias (INV-200300/0012).

\section{BIBLIOGRAFÍA}

1. Hoffmann JP. The effects of family relations on adolescent marijuana use. Int J Addict 1995; 30: 1207-1241.

2. Merikangas KR, Dierker L, Fenton B. (1998). Familial factors and substance abuse: Implications for prevention. En Ashery RS, Robertson EB, Kumpfer KL. (Eds.). Drug abuse prevention through family interventions. NIDA Research Monograph no. 177, 12-41. Rockville, MD: National Institute on Drug Abuse, 1998.

3. Molina BS, Chassin L, Curran PJ. A comparison of the mechanisms underlying substance use in early adolescent children of alcoholics and controls. J Stud Alc 1994; 55: 269-275.

4. Pons J. Familia y drogas: Una reflexión desde la psicología social. Rev Esp Drogodep 1994; 19: 279-280.

5. Pons J, Berjano E. El consumo abusivo de alcohol en la adolescencia: Un modelo explicativo desde la psicología social. Madrid: Plan Nacional sobre Drogas, 1999.

6. Pons J, Buelga S. Familia y conductas desviadas: el consumo de alcohol. En: Musitu G, Allat P. (Eds.). Psicosociología de la familia. 249-271. Valencia: Albatros, 1994.
7. Campins M, Gasch J, Hereu P, Rosselló J, Vaqué J. Consumo y actitudes de los adolescentes frente a sustancias adictivas: Encuesta de prevalencia. An Pediatr 1996; 45: 475-478.

8. Charro B, Martínez MP. Dinámica personal y familiar de los toxicómanos. Madrid: Universidad Pontificia de Comillas, 1995.

9. García-Pindado G. Determinantes familiares del consumo adolescente de droga, factores ambientales y genéticos. Psiquis 1992; 13: 413-422.

10. Pérez A, Mejía IE. Patrones de interacción de familias en las que no hay consumidores de sustancias psicoactivas. Adicciones 1998; 10: 111-119.

11. Elzo J, Ayestarán S, García N, González de Audicana M, Laespada MT, Vega A, Zulueta M. Drogas y Escuela V. Vitoria-Gasteiz: Servicio Central de Publicaciones Gobierno Vasco, 1996.

12. Pardeck JT. A multiple regression analysis of family factors affecting the potential for alcoholism in college students. Fam Ther 1991; 18: 115-121.

13. Protinsky $H$, Shilts L. Adolescent substance use and family cohesion. Fam Ther 1990, 17: 173-175.

14. Jurich HP, Polson CJ. Family factors in the lives of drug users and abusers. Adolescence 1985; 77: 143-159.

15. Tarter RE, Blackson T, Martin CH, Loebe, R, Moos HB. Characteristics and correlates of child discipline practices in substance abuse and normal families. Am J Addict 1993; 2: 18-25.

16. Kandel DB. Progression to regular marijuana involvement, phenomelogy and risk factors for near-daily use. En: Glantz M, Pickens R. (eds.). Vulnerability to drug abuse, 211-253. Washington DC. American Psychological Association, 1992.

17. Barnea Z, Teichman M, Rahav G. Personality, cognitive and interpersonal factors in adolescent substance use. Longitudinal test of an integrative model. J Youth Adolesc 1992; 21: 187-201.

18. Cancrini L. La psicoterapia: gramática y sintaxis. Barcelona: Paidos, 1991.

19. Sanz M. El funcionamiento familiar de los drogodependientes a lo largo de un tratamiento. Tesis doctoral. Bilbao, Universidad de Deusto, 2002.

20. Vielva I, Pantoja L, Abeijón JA. El funcionamiento de la familia con hijos (consumidores y no consumidores) de comportamientos no problemáticos. Bilbao: Universidad de Deusto, 2001.

21. Ayerbe A, Espina A, Pumar B, García E, Santos A. Clima familiar y características sociodemográficas en familias de toxicómanos. Adicciones 1997; 9: 375-390.

22. Kornblit $A L$, Mendes $A M$. Recaídas en drogadicción: factores de riesgo. Rev Esp Drogodep 1990; 2: 97-109.

23. Otero JM, Mirón L, Luengo A. Influence of family and peer group on the use of drugs by adolescents. Int J Addict 1989; 24: 1065-1082. 
24. Salazar I, Rodríguez A. Conflicto y dinámica familiar. Comunicación Psiquiátrica. An Univ Esp Psiquiatr 1994; XVIII: 217-239.

25. Bragado C, Bersabé R, Carrasco F. Factores de riesgo para los trastornos conductuales, de ansiedad, depresivos y de eliminación en niños y adolescentes. Psicothema 1999; 11: 939-956.

26. Buehler C, Gerard JM. Marital conflict, ineffective parenting and children's and adolescents' maladjustment. J Marriage Fam 2002; 64: 78-92.

27. Davies P, Harold G, Goeke-Morey M, Cummings E. Child emotional security and interparental conflict. Monographs of the Society for Research in Child Development 2002; 67 (3): vii-viii.

28. Dukewich TL. Examining affect and cognition as mechanisms underlying the marital conflict-child adjustment relationship. Dissertation Abstracts International: Section B: the Sciences \& Engineering 2001; 62 (3-B): 1615.

29. Katz LF. Physiological processes and mediators of the impact of marital conflict on children. En: Grych JH, Fincham FD. (eds.). Interparental conflict and child development: Theory, research and applications, 188212. Seattle WA: Universidad de Washington, 2001.

30. Mirón L, Luengo A, Sobral J, Otero JM. Un análisis de la relación entre ambiente familiar y delincuencia juvenil. Rev Psicol Social 1988; 3: 165-180.

31. Sigda KB. Conduct problems among younger siblings of delinquent adolescents: Relationship quality and delinquency consequences as contexts for learning processes. Dissertation Abstracts International: Section B: the Sciences \& Engineering 2000; 60 (7-B): 3579.

32. Kelly J. Children's Adjustment in conflicted marriage and divorce: A decade review of research. J Am Acad Child Adolesc Psychiatry 2000; 39: 963-973.

33. Malkus BM. Family dynamic and structural correlates of adolescent substance abuse: A comparison of families of non- substance abusers and substance abusers. J Child Adolesc Subst Abuse 1994; 3: 39-52.

34. Ruiz P, Lozano E, Polaino-Lorente A. Variables personales, familiares y patrones de consumo de alcohol y drogas ilegales en el adolescente. An Psiquiatr 1994; 10: 157162.

35. Muñoz-Rivas MJ, Graña JL. Factores familiares de riesgo y de protección para el consumo de drogas en adolescentes. Psicothema 2001; 13: 87-94.

36. Fincham FD, Grych JH, Osborne LN. Does marital conflict cause child maladjustment? Directions and challenges for longitudinal research. J Fam Psychol 1994; 8: 128140.

37. Kaplan JA. Family relationships and parent-child alliances: Their role in shaping the connections between parents' marriage and children's adaptations. Dissertation Abstracts International: Section B: the Sciences \& Engineering 2001; 61 (7-B): 3848.
38. Grych JH, Seid M, Fincham FD. Assessing Marital Conflict From The Child's Perspective: The Children's Perception Of Interparental Conflict Scale. Child Development 1992; 63: 558-572.

39. Grych JH, Fincham FD. Marital conflict and children's adjustment: a cognitive-contextual framework. Psychol Bull 1990; 108: 267-290.

40. Martínez-Pampliega A, Sanz M, Iraurgi I, et al. Impacto de la ruptura matrimonial en el bienestar físico y psicológico de los hijos(as). Estudio longitudinal en familias monoparentales a cargo de la madre. Primer informe, adecuación de los instrumentos y datos del estudio piloto. Universidad de Deusto-Emakunde, Bilbao, 2004.

41. Schaefer E. Child's Reports of Parental Behavior: An Inventory. Child Development 1965; 36: 413-424.

42. Mestre MV, Samper P, Tur A, Díez I. Estilos de crianza y desarrollo prosocial de los hijos. Rev Psicol Gen Apl 2001; 54: 4, 691-703.

43. Olson DH, Russell CS, Sprenkle DH. Circumplex model of marital and family system: VITheoretical update. Fam Process 1983; 22: 69-83

44. Olson DH, Portner J, Lavee Y. Faces III. St. Paul: University of Minnesota, 1985.

45. Olson DH, Russell CS, Sprenkle DH. Circumplex model: systematic assessment and treatment of families. New York: The Haworth Press, 1988.

46. Olson DH, Tiesel JW, Gorall DM, Fitterer C. Family Assessment Package. St. Paul MN, University of Minnesota, 1996.

47. Hudson WW. The clinical measurement package. A field manual. Chicago: Dorsey Press, 1982.

48. Hudson WW. The WALMYR assessment scales scoring manual. WALMYR Publishing: Tempe, 1992.

49. Booth A, Edwards J. Measuring marital instability. J Marriage Fam 1983; 45: 387-393.

50. Marañón M, Oquiñena J, Aguirre L. Euskadi y Drogas 1998. Vitoria-Gasteiz: Servicio de Publicaciones de Gobierno Vasco, 1999.

51. Sánchez-Cantalejo E. Regresión logística en salud pública. Escuela Andaluza de Salud Publica, Serie Monografías № 26. Granada: EASP; 2000.

52. Laespada T, Arostegi E, Elzo J, García-del-Moral N, Iraurgi I. (2005). Euskadi y Drogas 2004. Vitoria-Gasteiz: Servicio Central de Publicaciones del Gobierno Vasco.

53. Katz LF, Gottman JM. (1993). Patterns of marital conflict predict children's internalizing and externalizing behaviours. Develop Psychol 1993; 29: 940-950.

54. Secades R, Fernández-Hermida JR. Intervención familiar en la prevención de las drogodependencias. En Plan Nacional sobre Drogas (Ed.), Factores de riesgo familiares para el uso de drogas: Un estudio empírico español (pp. 58-113). Madrid: Plan Nacional de Drogas, 2001.

55. Guttman L. What is not what statistics. Statistician 1977; 26: 81-107. 